\title{
Hidden Quantum Groups Inside Kac-Moody Algebra
}

\author{
A. Alekseev, L. Faddeev, and M. Semenov-Tian-Shansky \\ St. Petersburg Branch of Steklov Mathematical Institute, Fontanka 27, \\ St. Petersburg 191011, Russia
}

Received September 1, 1991

\begin{abstract}
A lattice analogue of the Kac-Moody algebra is constructed. It is shown that the generators of the quantum algebra with the deformation parameter $q=\exp (i \pi / k+h)$ can be constructed in terms of generators of the lattice KacMoody algebra (LKM) with the central charge $k$. It appears that there exists a natural correspondence between representations of the LKM algebra and the finite dimensional quantum group. The tensor product for representations of the LKM algebra and the finite dimensional quantum algebra is suggested.
\end{abstract}

\section{Introduction}

Fascinating links between conformal field theory and quantum groups discovered recently suggest that quantum groups also have a direct bearing on the representation theory of Kac-Moody algebras. It is the purpose of the present note to trace down this hidden quantum group symmetry in the framework of KacMoody algebras. Our main result is that the monodromy of quantum Kac-Moody current when properly regularized satisfies the commutation relations of the quantized universal enveloping algebra $U_{q}(\mathfrak{g})$ with $q$ related to the central charge $k$ via $q=\exp \left(\frac{\pi i}{k+n}\right)$. The regularized definition of the monodromy is based in its turn on a lattice version of the current algebra which we also describe in this paper. This algebra associated with a periodic 1-dimensional lattice is already quantum (i.e. incorporates parameter $q$; in fact, it is defined for any $q$, not only for roots of unity) and also takes into account the central charge. It may be regarded as a nontrivial deformation of $U_{q}(\mathfrak{g})^{\otimes N}$. (The very existence of such deformations is a typically quantum phenomenon. Indeed, it is well known that classical semisimple Lie groups and Lie algebras are rigid. By contrast, quantum universal enveloping algebras admit certain deformations which may be regarded as finitedimensional counterparts of central extensions of current algebras.) Our first key result is the monodromy theorem for this lattice algebra which asserts that the 
monodromy satisfies the commutation relations of $U_{q}(\mathfrak{g})$. In the scaling limit the lattice current algebra becomes the ordinary classical current algebra with fixed central charge, while the monodromy remains quantum. This result has several important corollaries. First, we are able to describe the regularized Casimir operators for the Kac-Moody algebra and to relate them to the quantum Casimir operators for $U_{q}(\mathrm{~g})$. Second, we define for the lattice algebra the structure of a $U_{q}(\mathrm{~g})$-comodule. This structure reproduces the well known tensor product properties of $U_{q}(\mathfrak{g})$. Remarkably, it essentially coincides with the fusion rules in conformal field theory.

The present note represents a part of the research program now in progress. Our approach combines ideas from different independent sources. We should mention the papers $[1,2]$ which lead to the definition of lattice current algebras and the papers [3-7] on quantum exchange algebras. Some aspects of our results were reported in [8]. While for the lattice current algebras our results are completely rigorous, the details of our construction in the scaling limit are still to be worked out. In this note we content ourselves with the physical level of rigour. Complete proofs and the mathematical background of the construction of lattice current algebras will be given in a subsequent paper.

\section{Current Algebras: A Brief Reminder}

Let $\mathfrak{g}$ be a finite dimensional simple Lie algebra, $\tilde{\mathfrak{g}}$ the associated current algebra on the circle. For simplicity, we may assume that $\mathfrak{g}=s l(2, \mathbb{C})$. By definition, the current algebra with central charge $k$ is a Lie algebra with generators $J^{a}(x), 0 \leqq x \leqq 2 \pi$, associated with an orthogonal basis $\left\{\sigma^{a}\right\}$ in $\mathfrak{g}$, satisfying the commutation relations

$$
\left[J^{a}(x), J^{b}(y)\right]=f_{c}^{a b} J^{c}(x) \delta(x-y)+\frac{i k}{2 \pi} \delta^{a b} \delta^{\prime}(x-y),
$$

where $f_{c}^{a b}$ are the structure constants of $g$ with respect to the basis $\left\{\sigma^{a}\right\}$. To get the more familiar generators one has to perform the formal Fourier transform

$$
J^{a}(x)=\frac{1}{2 \pi} \sum_{k \in \mathbb{Z}} e^{i k x} J_{k}^{a} .
$$

Then (1) is equivalent to

$$
\left[J_{m}^{a}, J_{n}^{b}\right]=f_{c}^{a b} J_{m+n}^{c}+k m \delta^{a b} \delta_{m+n, 0} .
$$

We are interested in representations of the algebras (1) which may be integrated to projective unitary representations of the corresponding compact loop group. This means that if the generators $J^{a}(x)$ correspond to the orthogonal basis of the compact real form of $\mathfrak{g}$, the constant $i k$ should be integer and purely imaginary. We shall see below that passing to the compact real form of the lattice current algebra defined in the next section presents some difficulties, so we have to deal with complex algebras while still keeping the natural condition on the central charge. It is convenient to suppress the Lie algebra indices in (1). Let us put

$$
J(x)=J^{a}(x) \sigma_{a}, \quad C=\sigma_{a} \otimes \sigma_{a} .
$$

Then (1) is rewritten as

$$
\left[J_{1}(x), J_{2}(y)\right]=\frac{1}{2}\left[C, J_{1}(x)-J_{2}(y)\right] \delta(x-y)+\frac{i k}{2 \pi} C \delta^{\prime}(x-y),
$$


where the indices 1,2 refer to two copies of the auxiliary space $\mathfrak{g}$ and $J_{i}(x)$ is regarded as an element of $\tilde{\mathfrak{g}} \otimes \mathfrak{g}_{i} \subset \tilde{\mathfrak{g}} \otimes \mathfrak{g}_{1} \otimes \mathfrak{g}_{2}$. The commutation relations (3) are invariant with respect to the action of gauge transformations

$$
J(x) \mapsto g(x) J(x) g(x)^{-1}+\frac{i k}{2 \pi} \partial_{x} g \cdot g^{-1}(x),
$$

and in particular with respect to global gauge transformations

$$
J(x) \mapsto g J(x) g^{-1} .
$$

The monodromy of the current $J(x)$ is formally defined by

$$
M=P \exp \left(\frac{2 \pi i}{k} \int_{0}^{2 \pi} J(x) d x\right) .
$$

Since the commutation relations for $J(x)$ are highly singular, it is not easy to derive the commutation relations for $M$. One of our main assertions is that when properly regularized $M$ has the commutation relations of the quantum universal enveloping algebra $U_{q}(\mathfrak{g})\left[\right.$ with $q$ related to central charge $k$ by $\left.q=\exp \left(\frac{\pi i}{k+n}\right)\right]$. This may be regarded as a new type of anomaly which leads to the spontaneous breakdown of the global gauge symmetry (5). [There is some evidence that this anomaly is associated with the cohomology class in $H^{3}(\mathrm{~g})$ which also generates via transgression the Schwinger anomaly in the commutation relations (3) - see discussion in Sect. 5 below.]

The usual definition of the quantized universal enveloping algebra $U_{q}(\mathfrak{g})$ is by means of generators and relations. (For $\mathrm{g}=s l(2)$ it is due to Kulish, Reshetikhin, and Sklyanin, while the general case was worked out by Drinfeld and Jimbo (see [9]).) As an algebra, $U_{q}(s(2))$ is generated by the elements $H, X_{+}, X_{-}$which satisfy the following relations

$$
\begin{gathered}
{\left[H, X_{ \pm}\right]= \pm 2 X_{ \pm},} \\
{\left[X_{+}, X_{-}\right]=\frac{\sinh (h H)}{\sinh (h)} .}
\end{gathered}
$$

The coalgebra structure which determines the tensor product of the representations is given by

$$
\begin{aligned}
& \Delta H=H \otimes 1+1 \otimes H, \\
& \Delta X_{ \pm}=e^{\frac{h H}{2}} \otimes X_{ \pm}+X_{ \pm} \otimes e^{-\frac{h H}{2}} .
\end{aligned}
$$

There is a different way to express these commutation relations, due to Faddeev, Reshetikhin, and Takhtajan [10]. Let $R=R(q)$ be the quantum $R$-matrix associated with the standard representation of $U_{q}(\operatorname{sl}(2))$ in $\mathbb{C}^{2}, R \in \operatorname{End}\left(\mathbb{C}^{2} \otimes \mathbb{C}^{2}\right)$ :

$$
R=\left(\begin{array}{cccc}
q & 0 & 0 & 0 \\
0 & 1 & 0 & 0 \\
0 & q-q^{-1} & 1 & 0 \\
0 & 0 & 0 & q
\end{array}\right), \quad q=e^{h}
$$


The $R$-matrix (9) satisfies the quantum Yang-Baxter identity

$$
R_{12} R_{13} R_{23}=R_{23} R_{13} R_{12} .
$$

Along with $R$ we shall also need the matrices

$$
R^{+}=P R P, \quad R^{-}=R^{-1} .
$$

[Here $P \in \operatorname{End}\left(\mathbb{C}^{2} \otimes \mathbb{C}^{2}\right)$ is the permutation matrix defined by $(P(x \otimes y)=y \otimes x$. $]$ Consider the $2 \times 2$-matrices $L^{+}, L^{-}$whose coefficients are generators of $\left.U_{q}(s)(2)\right)$ :

$$
L^{+}=\left(\begin{array}{cc}
q^{-H} & -\left(q-q^{-1}\right) X_{+} \\
0 & q^{H}
\end{array}\right), \quad L^{-}=\left(\begin{array}{cc}
q^{H} & 0 \\
\left(q-q^{-1}\right) X_{-} & q^{-H}
\end{array}\right) .
$$

Then the following commutations are satisfied

$$
\begin{aligned}
& L_{1}^{ \pm} L_{2}^{ \pm} R^{+}=R^{+} L_{2}^{ \pm} L_{1}^{ \pm}, \\
& L_{1}^{+} L_{2}^{-} R^{+}=R^{+} L_{2}^{-} L_{1}^{+} .
\end{aligned}
$$

It is convenient to combine $L^{+}, L^{-}$into a single matrix $L=L^{+}\left(L^{-}\right)^{-1}$. The commutation relations for $L$ are

$$
\left(R^{-}\right)^{-1} L_{1} R^{-} L_{2}=L_{2}\left(R^{+}\right)^{-1} L_{1} R^{+} .
$$

One can show that a free associative algebra generated by the matrix coefficients of $L=\left(L_{i j}\right)$ satisfying the relations (14) is isomorphic to $U_{q}(s l(2))$. The rearrangement of simple commutation relations ( 7$)$ into the rather complicated form $(13,14)$ may seem artificial. However, there are profound mathematical reasons to prefer this form of commutation relations. In a sense, passing from (7) to (14) or (13) is similar to passing from a Lie algebra to the corresponding Lie group. We shall not go into further discussion of this point (although a complete exposition of the underlying theory is still lacking in the existing literature), but simply notice that the commutation relations for the monodromy (6) have exactly the form (14). Another important fact is that relations (14) serve as a starting point to define the lattice version of the current algebra.

Let us finally discuss the coproduct structure and the tensor products in terms of the generators $L^{ \pm}, L$. Assume that there are two representations of $U_{q}(\mathfrak{g})$ in linear spaces $\mathscr{H}^{\prime}, \mathscr{H}^{\prime \prime}$. Let $L^{\prime}, L^{\prime \prime}$ be the matrices of generators acting in $\mathscr{H}^{\prime}, \mathscr{H}^{\prime \prime}$, respectively. Put

$$
\Delta L=\left(L^{+}\right)^{\prime} L^{\prime \prime}\left(\left(L^{-}\right)^{-1}\right)^{\prime} .
$$

We may regard $\Delta L$ as a $2 \times 2$-matrix whose coefficients are linear operators acting in $\mathscr{H}^{\prime} \otimes \mathscr{H}^{\prime \prime}$. Then $\Delta L$ also satisfies commutation relations (14) and hence defines a representation of $U_{q}(\mathrm{~g})$ in $\mathscr{H}^{\prime} \otimes \mathscr{H}^{\prime \prime}$. One can show that this construction agrees with the standard definition of the tensor product for $U_{q}(\mathrm{~g})$ based on (8).

\section{Lattice Version of Current Algebras}

As already explained in the Introduction, our strategy will be as follows. First, we describe the lattice version of commutation relation (3) and state the corresponding monodromy theorem. Then we discuss the continuous limit. 
By definition, the lattice current algebra $\mathscr{A}_{L C}$ is a free algebra generated by matrix coefficients of $2 \times 2$-matrices $L^{i}, i=1,2, \ldots, N$, satisfying the following relations

$$
\begin{aligned}
L_{1}^{i} L_{2}^{i} & =R^{+} L_{2}^{i} L_{1}^{i} R^{-}, \\
L_{1}^{i} R^{-} L_{2}^{i-1} & =L_{2}^{i-1} L_{1}^{i}, \\
L_{1}^{i} L_{2}^{j} & =L_{2}^{j} L_{1}^{i} \text { for }|i-j| \geqq 2 .
\end{aligned}
$$

We assume that $i+N \equiv i$, i.e. that the matrices $L^{i}$ are associated with a periodic 1-dimensional lattice. To make these relations more transparent let us introduce the $R$-matrix on the lattice

$$
R_{i-j}^{ \pm}= \begin{cases}R^{ \pm}, & i=j, \\ I, & i \neq j .\end{cases}
$$

Commutation relations (14) for $U_{g}(\mathfrak{g})^{\otimes N}$ may be rewritten in the form

$$
\left(R_{i-j}^{-}\right)^{-1} L_{1}^{i} R_{i-j}^{-} L_{2}^{j}=L_{2}^{j}\left(R_{i-j}^{+}\right)^{-1} L_{1}^{i} R_{i-j}^{+} .
$$

The relations (16) have the form

$$
\left(R_{i-j}^{-}\right)^{-1} L_{1}^{i} R_{i-j+1}^{-} L_{2}^{j}=L_{2}^{j}\left(R_{i-j-1}^{+}\right)^{-1} L_{1}^{i} R_{i-j}^{+} .
$$

In other words, the perturbation of the relations (14) consists in replacing conjugation by $R^{ \pm}$with lattice gauge transformations (cf. [2] where similar commutation relations are derived for central extensions of quantum Kac-Moody algebras). We omit the mathematical background which serves as a motivation for this definition. (Its classical counterpart is already present in [1].) Relations (18) are not ultralocal in that the neighbouring matrices $L^{i}, L^{i \pm 1}$ do not commute with each other. In [8] a change of variables is described which replaces (18) with local relations. This change of variables is important for the study or representations of $\mathscr{A}_{L C}$. We are planning to discuss these questions in a separate publication. The main property of the lattice current algebra is given by the following theorem.

Theorem. Put

$$
M=L_{N} L_{N-1} \ldots L_{2} L_{1}
$$

Then the monodromy matrix $M$ satisfies relations (14).

The commutation relations of $M$ with $L_{i}$ is given by

$$
M_{1} R_{i}^{-} L_{2}^{i}\left(R_{i}^{-}\right)^{-1}=L_{2}^{i}\left(R_{i-1}^{+}\right)^{-1} M_{1} R_{i-1}^{+} \text {. }
$$

One may notice that the definition of $M$ depends on the initial point $k=1$. In a similar way we may define monodromies

$$
M_{k}=\prod_{i=k}^{k+N-1} L_{i}
$$

which satisfy (14) and also

$$
M_{1}^{k} R_{i-k+1}^{-} L_{2}^{i}\left(R_{i-k+1}^{-}\right)^{-1}=L_{2}^{i}\left(R_{i-k}^{+}\right)^{-1} M_{1}^{k} R_{i-k}^{+} .
$$

Commutation relations (18) admit certain quantum automorphisms. To describe them let us first recall the definition of the quantum group (as opposed to the 
quantum universal enveloping algebra). By definition, the algebra $A=\operatorname{Fun}_{q}(G)$ is a free algebra with generators $T=\left(T_{i j}\right)$ and relations

$$
T_{1} T_{2} R^{+}=R^{+} T_{2} T_{1} \text {. }
$$

For various classical groups these relations are supplemented by the symmetry relations for $T$ and the condition on its determinant. For instance, for $G=S L(2)$ the latter relation has the form

$$
\operatorname{det}_{q} T=T_{11} T_{22}-q T_{12} T_{21}=1 \text {. }
$$

One can show that as a-Hopf algebra $A$ is the dual of $U_{q}(\mathrm{~g})$. The duality is set up by

$$
\left\langle L^{ \pm}, T\right\rangle=\left(R^{ \pm}\right)^{-1} .
$$

Now let $A^{\otimes N}=A \otimes A \otimes \ldots \otimes A$ and put

$$
\tilde{L}^{i}=T^{i+1} L^{i}\left(T^{i}\right)^{-1}
$$

where we assume that $L^{i}$ and $T^{j}$ commute with each other. Then $\tilde{L}^{i}$ satisfies the same relations (18). [In more mathematical terms, (26) defines the structure of an $A^{N}$-comodule on $\mathscr{A}_{L C}$.]

Another interesting question is the structure of the Casimir operators of $\mathscr{A}_{L C}$. Theorem. For generic $q$ and $N$ odd the center of $\mathscr{A}_{L C}$ is a free algebra generated by 1 and the elements

$$
C_{k}=\operatorname{tr} q^{2 e} M^{k}, \quad k=1,2, \ldots, \text { rankg. }
$$

(Here $\varrho$ is half the sum of positive roots.)

It is well known that $C_{k}$ generate the center of $U_{q}(\mathfrak{g})$. (This was proved by [10], cf. also $[11,12]$.)

In more mathematical terms we may regard the monodromy map as an embedding

$$
M^{*}: U_{q}(\mathfrak{g}) \hookrightarrow \mathscr{A}_{L C} \text {. }
$$

The theorem then asserts that the extension

$$
\operatorname{cent} U_{q}(\mathfrak{g}) \hookrightarrow U_{q}(\mathfrak{g}) \hookrightarrow \mathscr{A}_{L C}
$$

is central. The semiclassical version of this theorem was proved in [1]. Along with the monodromy (19) we shall also need the wave functions

$$
u^{i}=\prod_{1 \leqq k \leqq i} L_{i}
$$

which satisfy the following exchange relations:

$$
u_{1}^{i} u_{2}^{j}=u_{2}^{j} u_{1}^{i} R^{+}, \quad i>j, \quad u_{1}^{i} u_{2}^{j}=u_{2}^{j} u_{1}^{i} R^{-}, \quad i<j .
$$

The exchange algebra (29) will be used in Sect. 5 to derive the scaling limit. Relations at one point have the form

$$
u_{1}^{i} u_{2}^{i}=R^{+} u_{2}^{i} u_{1}^{i} R^{-} .
$$

\section{Lattice Current Algebra as a Comodule}

It is possible to define an action of $\mathscr{A}_{L C}$ in tensor products of representation spaces of $\mathscr{A}_{L C}$ with representation spaces of $U_{q}(\mathrm{~g})$. Let $\mathscr{H}$ be a representation space of $\mathscr{A}_{L C}$ 
and $V$ a' representation space for $U_{q}(\mathfrak{g})$. We denote by $\left(L^{i}\right)^{\prime}$ the matrices of generators of $\mathscr{A}_{L C}$ acting in $\mathscr{H}$ and by $\left(N^{ \pm}\right)^{\prime \prime}$ the matrices of generators of $U_{q}(\mathrm{~g})$ satisfying relations (9) which act in $V$.

We define matrices $\tilde{L}^{i}$ acting in $\mathscr{H} \otimes V$ by

$$
\begin{aligned}
\widetilde{L}^{i} & =\left(L^{i}\right)^{\prime}, \quad i \neq 1, N, \\
\tilde{L}^{1} & =\left(L^{1}\right)^{\prime}\left(\left(N^{-}\right)^{-1}\right)^{\prime \prime}, \\
\tilde{L}^{N} & =\left(N^{+}\right)^{\prime \prime}\left(L^{N}\right)^{\prime} .
\end{aligned}
$$

It is easy to check that matrices $\tilde{L}^{i}$ satisfy relations (18). In more mathematical terms, we may regard the mapping $C: L^{i} \rightarrow \widetilde{L}^{i}$ as a homomorphism from $\mathscr{A}_{L C}$ into $\mathscr{A}_{L C} \otimes U_{q}(\mathfrak{g})$. Clearly, it satisfies

$$
(C \otimes \mathrm{id}) C=(\mathrm{id} \otimes \Delta) C
$$

and hence is consistent with the coproduct $\Delta$ in $U_{q}(\mathrm{~g})$. The monodromy $M(L)$ is mapped into $\left(N^{+}\right)^{\prime \prime} M(L)^{\prime}\left(\left(N^{-}\right)^{-1}\right)^{\prime \prime}$, and hence we have a commutative diagram

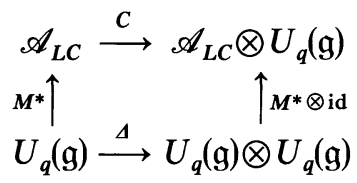

which expresses consistency with the monodromy map.

The comodule structure of $\mathscr{A}_{L C}$ suggests simple tensor properties of its representation. First of all, since $\mathscr{A}_{L C}$ and $U_{q}(\mathrm{~g})$ have common Casimir operators, it seems plausible that an irreducible representation $\mathscr{H}_{s}$ of $\mathscr{A}_{L C}$ is uniquely specified by its "spin" $s$. Moreover, as a $U_{q}(\mathrm{~g})$-module the space $\mathscr{H}_{s}$ decomposes into direct sum of "isotypical" components with the same spin $s$. Thus

$$
\mathscr{H}_{s}=\mathscr{H} \otimes V_{s},
$$

where the monodromy acts trivially in $\mathscr{H}$. We then have

$$
\mathscr{H}_{s} \otimes V_{j} \simeq\left(\mathscr{H} \otimes V_{s}\right) \otimes V_{j} \simeq\left(\mathscr{H} \otimes V_{j}\right) \otimes V_{s},
$$

and hence (by the uniqueness assumption)

$$
\mathscr{H}_{s} \otimes V_{j} \simeq \mathscr{H}_{j} \otimes V_{s} .
$$

Moreover, the decomposition into irreducible representations of $\mathscr{H}_{s} \otimes V_{j}$ and its braiding properties are completely determined by the corresponding properties of $U_{q}(\mathfrak{g})$. Clearly, this picture resembles the fusion algebra in conformal field theory. The tensor algebra still makes sense if $q$ is a root of unity (the latter condition being imposed by the scaling properties of our algebra, as explained in the next section).

\section{The Scaling Limit}

Let us now discuss the continuous limit of the lattice current algebra. We shall argue that the commutation relations for currents become those of the ordinary Kac-Moody algebra, while the commutation relations for the monodromy remain quantum. As mentioned in the Introduction our arguments will be not completely 
rigorous. The starting point is the exchange algebra (29). If we replace the lattice variable $i$ with the continuous variable $x$ we get the following exchange algebra

$$
u_{1}(x) u_{2}(y)=u_{2}(y) u_{1}(x) R(x-y),
$$

where

$$
\begin{aligned}
R(x-y) & =R^{+} \theta(x-y)+R^{-} \theta(y-x), \\
\theta(x) & = \begin{cases}1, & x>0 \\
0, & x<0\end{cases}
\end{aligned}
$$

For $x=y$ we get formally

$$
u_{1}(x) u_{2}(x)=R^{+} u_{2}(x) u_{1}(x) R^{-},
$$

but this relation has, of course, a dubious status since it does not survive when $u_{1}$ and $u_{2}$ are smoothed down by averaging with test functions.

The current $J(x)$ is formally the derivative of $u(x)$,

$$
J(x)=\partial_{x} u \cdot u^{-1} .
$$

However, since the commutator of $u$ 's has singularities at coinciding points we must take care to extract them first. Thus we write

$$
\frac{1}{\varepsilon} u^{\prime}(x+\varepsilon) u(x)^{-1}=\frac{A}{\varepsilon}+J(x)+O(\varepsilon) .
$$

By dimension count the singular term $A / \varepsilon$ is the only one possible. For symmetry reasons, the matrix $A$ is scalar, $A=a I$. We may also write

$$
u_{1}^{\prime}(x+\varepsilon) u_{2}(x)^{-1}=\frac{B}{\varepsilon}+\text { regular terms }
$$

where $B$ is a matrix in the tensor square. Again for symmetry reasons, $B_{i j k l}=b \delta_{j k} \delta_{i l}$. A simple calculation shows that $a=n b$, where $n$ is the size of our matrices.

The relation of the current $J$ to the lattice variables is formally

$$
L^{i}=P \exp \int_{x}^{x+\varepsilon} J(y) d y .
$$

Thus we expect that in the scaling limit lattice variables are close to identity. A more thorough inspection shows, however, that this is true only for normal ordered exponentials

$$
: L^{i}:=: P \exp \int_{x}^{x+\varepsilon} J(y) d y: \underset{\varepsilon \rightarrow 0}{\longrightarrow} I .
$$

The lattice variables $L^{i}$ differ from these normal ordered exponentials by a finite factor

$$
L^{i}=e^{a}: L^{i},
$$

where $a$ is the same constant as in (36). Below we shall actually compute this constant using the operator expansion. The computation of the commutation relations for currents which follow from the exchange algebra (33) is based on the following general formula for the $R$-matrices

$$
R^{ \pm}=F_{12} q^{ \pm P} F_{21}^{-1},
$$


where $P$ is the permutation matrix, $F_{12}$ an invertible matrix in $\mathbb{C}^{n} \otimes \mathbb{C}^{n}$, and $F_{21}=P F_{12} P$. Hence we have for the $R$-matrix $R(x-y)$ :

$$
R(x-y)=F_{12} q^{P \operatorname{sign}(x-y)} F_{21}^{-1} .
$$

Formulae (41), (42) are based on the general philosophy of "quasi-Hopf algebras," due to Drinfeld [13]. Before proceeding to the actual computation it is worth commenting on the meaning of these formulae and their general implications. As already noted, the regularized commutation relations for the monodromy are the manifestation of symmetry breaking which introduces quantum $R$-matrices into the basic commutation relations. Now, for higher rank groups the quantization and hence the $R$-matrices are certainly not unique, and one might wonder why the particular $R$-matrix which defines the theory is preferred to all the others. According to the general philosophy of Drinfeld [13], the uniqueness of quantization is restored, modulo some natural equivalence relation, in a larger class of algebras for which the coassociativity constraint is replaced by a milder assumption. Formula (41) shows, in particular, that any quantum $R$-matrix is "gauge equivalent" to the standard $R$-matrix

$$
R_{0}=q^{P} .
$$

(More precisely, this is true for a certain class of $R$-matrices which define the socalled quasi-triangular Hopf algebras; all physically relevant $R$-matrices fall within this class.)

The permutation operator $P$, or, more generally, the Casimir operator $C$, is stable, i.e. it does not depend on the particular choice of quantization. [One might show that this stability has a cohomological nature: the Casimir operator defines an element in $H^{3}(\mathrm{~g})$ which may be regarded as the cohomology class of quantization. Incidentally, this same cohomology class also determines, via transgression, the cohomology class of the central extension of the current algebra.] Of course, it would be conceptually more simple to work directly with the $R$-matrix (43), but as explained in [13] this leads to complications with associativity.

Our general conclusion resolving the difficulty referred to above is that one may take any $R$-matrix in the given "gauge class" and this will lead to essentially equivalent results (choosing an $R$-matrix means roughly to specify some particular regularization of the theory). The independence of the choice of $R$ is manifested in the computation of the Schwinger commutation relation for currents: as we shall see, the answer depends only on $P$, and the "gauge matrix" $F_{12}$ is cancelled out.

Let us now proceed to the actual computation. We shall compute the commutation relations between $J$ and $u$ in two ways, which will allow to fix the so far unspecified constant $a$. We have

$$
u_{1}^{\prime}(x+\varepsilon) u_{1}(x)^{-1} u_{2}(y)=u_{1}^{\prime}(x+\varepsilon) u_{2}(y) R(x-y)^{-1} u_{1}(x)^{-1} .
$$

Using the expansion (36) and the formula

$$
R^{+}(x-y-\varepsilon)^{-1}=R^{+}(x-y)^{-1}+2 \varepsilon \ln q \cdot F_{12} P F_{21}^{-1} \delta(x-y)+o(\varepsilon)
$$

we get, after simple calculation,

$$
\left[J_{1}(x), u_{2}(y)\right]=2 \ln q P u_{2}(y) \delta(x-y) .
$$


On the other hand, the expansion (37) implies that

$$
J_{1}(x) u_{2}(y)=-\frac{b}{x-y} P u_{2}(y)+\text { regular terms } .
$$

The operator expansion of the form

$$
A(x) B(y)=\frac{1}{x-y} C(y)+\text { regular terms }
$$

implies, by the Sokhotski-Plemel formula, that

$$
[A(x), B(y)]=2 \pi i C(y) \delta(x-y) .
$$

Thus a comparison of (45) and (46) yields

$$
b=-\frac{\ln q}{\pi i},
$$

and hence

$$
a=-\frac{n \ln q}{\pi i}
$$

Now we are ready to calculate the commutator of currents. We have

$$
\begin{aligned}
{\left[J_{1}(x), \frac{1}{\varepsilon} u_{2}^{\prime}(y+\varepsilon) u_{2}(y)^{-1}\right]=} & \frac{2 \ln q}{\varepsilon} P u_{2}^{\prime}(y+\varepsilon) u_{2}(y)^{-1} \delta(x-y-\varepsilon) \\
& -\frac{2 \ln q}{\varepsilon} u_{2}^{\prime}(y+\varepsilon) u_{2}(y)^{-1} P \delta(x-y) \\
& -\frac{2 \ln q}{\varepsilon} P u_{2}(y+\varepsilon) u_{2}(y)^{-1} \delta^{\prime}(x-y-\varepsilon) \\
= & 2 \ln q\left[P, J_{2}(y)\right] \delta(x-y)-2 \ln q(1+a) \delta^{\prime}(x-y) .
\end{aligned}
$$

and hence

$$
\left[J_{1}(x), J_{2}(y)\right]=2 \ln q\left[P, J_{2}(y)\right] \delta(x-y)-2 \ln q(1+a) P \delta^{\prime}(x-y) .
$$

Assume that $\ln q$ is purely imaginary,

$$
\ln q=\frac{\pi i}{k+n}
$$

Then

$$
\left[J_{1}(x), J_{2}(y)\right]=\frac{2 \pi i}{k+n}\left[P, J_{2}(y)\right] \delta(x-y)-2 \frac{2 \pi i k}{(k+n)^{2}} P \delta^{\prime}(x-y) .
$$

If we rescale the current by setting

$$
\widetilde{J}(x)=\frac{i(k+n)}{2 \pi} J,
$$

we get

$$
\left[\widetilde{J}_{1}(x), \widetilde{J}_{2}(y)\right]=\frac{1}{2}\left[P, J_{1}(x)-J_{2}(y)\right] \delta(x-y)+\frac{i k}{2 \pi} P \delta^{\prime}(x-y) .
$$


Thus we get

$$
\widetilde{J}(x)=\frac{i(k+n)}{2 \pi} \partial_{x} u \cdot u^{-1}
$$

Let us notice that the presence of $i=\sqrt{-1}$ in formula (53) for the rescaled current causes difficulties in dealing with real forms of our algebras. Indeed, (53) implies that $\widetilde{J}(x)$ is anti-Hermitian (i.e. lies in the compact form of the current algebra) if $u(x)$ is Hermitian. But in that case the monodromy

$$
M=P \exp \left\{-\frac{2 \pi i}{k+n} \int_{0}^{2 \pi} \tilde{J}(x) d x\right\}
$$

does not satisfy the expected unitary condition. The same trouble is also reflected by the fact that the quantization parameter $q=\exp \left(\frac{\pi i}{k+n}\right)$ is a root of unity while a bona fide $U_{q}(s u(n))$ is defined for real $q$. Of course, the emergence of quantum groups $U_{q}(\mathfrak{g})$ for $q$ a root of unity is highly typical for the problems of conformal field theory.

\section{References}

1. Semenov-Tian-Shansky, M.A.: Publ. RIMS 21 (6), 1237-1260 (1985), Kyoto Univ.

2. Reshetikhin, N.Yu., Semenov-Tian-Shansky, M.A.: Lett. Math. Phys. 19 (1990)

3. Babelon, O.: Phys. Lett. B 215, 523-527 (1988)

4. Blok, B.: Tel-Aviv University preprint (1989)

5. Alekseev, A., Shatashvili, S.: Commun. Math. Phys. 133, 353-368 (1990)

6. Faddeev, L.D.: Commun. Math. Phys. 132, 131-138 (1990)

7. Faddeev, L.D.: Lectures given in Schladming (1989)

8. Alekseev, A., Faddeev, L.D., Semenov-Tian-Shansky, M.A., Volkov, A.: The unravelling of the quantum group structure in the WZNW theory. Preprint CERN-TH-5981/91 (1991)

9. Drinfeld, V.: Quantum groups, pp. 798-820. Proc. ICM-86 Berkeley, California, USA, 1986, 1987

10. Faddeev, L.D., Reshetikhin, N.Yu., Takhtajan, L.A.: Algebra i Anal. 1 1, 178-206 (1989) (in Russian)

11. Drinfeld, V.: Algebra i Anal. 1 2, 30-47 (1989) (in Russian)

12. Reshetikhin, N.Yu.: Algebra i Anal. 1 2, 169-189 (1989) (in Russian)

13. Drinfeld, V.: Algebra i Anal. 1 6, 114-149 (1989) (in Russian)

Communicated by N. Yu. Reshetikhin 
